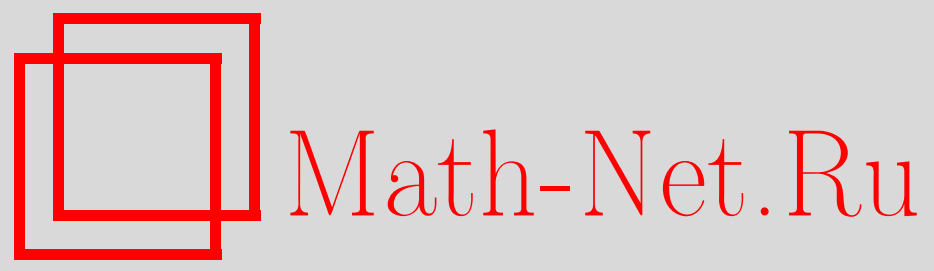

П. Н. Ярыкин, Устойчивость нелинейного стохастического процесса, аппроксимирующего систему взаимодействующих частиц, Теория вероятн. и ее примен., 2006, том 51, выпуск 2, 400-409

DOI: https://doi.org/10.4213/tvp63

Использование Общероссийского математического портала Math-Net.Ru подразумевает, что вы прочитали и согласны с пользовательским соглашением

http://www.mathnet.ru/rus/agreement

Параметры загрузки:

IP : 18.234 .197 .8

26 апреля 2023 г., 15:12:02

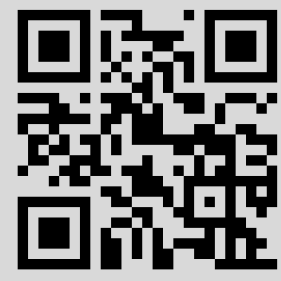


25. Nagaev S. V. On probability and moment inequalities for supermartingales and martingales. - Acta Appl. Math., 2003, v. 79, № 1-2, p. 35-46.

26. Rosenthal H. P. On the subspaces of $L^{p}(p>2)$ spanned by sequences of independent random variables. - Israel J. Math., 1970, v. 8, p. 273-303.

27. Burkholder D. L. Distribution function inequalities for martingales. - Ann. Probab., 1973 , v. 1, p. $19-42$.

28. Hitczenko $P$. Best constants in martingale version of Rosenthal's inequality. - Ann. Probab., 1990, v. 18, № 4, p. 1656-1668.

29. Hitczenko $P$. Upper bounds for the $L_{p}$-norms of martingales. - Probab. Theory Related Fields, 1990 , v. 86 , № 2, p. 225-238.

30. Ибрагимов P., Шарахметов III. О точной константе в неравенстве Розенталя. Теория вероятн. и ее примен., 1997, т. 42 , в. 2 , с. $341-350$.

31. Ибрагимов P., Шарахметов Ш. Точная константа в неравенстве Розенталя для случайных величин с нулевым средним. - Теория вероятн. и ее примен., 2001, т. 46 , в. 1 , c. $134-138$.

32. Пешкир $Г$., Ширяев $A . H$. Неравенства Хинчина и мартингальное расширение сферы их действия. - Успехи матем. наук, 1995, т. 50, № 5, с. 3-62.

33. Johnson W. B., Schechtman G., Zinn J. Best constants in moment inequalities for linear combinations of independent and exchangeable random variables. - Ann. Probab., 1985 , v. 13, № 1 , p. 234-253.

34. Нагаев С. В. Некоторые уточнения вероятностных и моментных неравенств. Теория вероятн. и ее примен., 1997, т. 42, в. 4, с. 832-838.

Поступила в редакцию

11.VI.2002

Исправленный вариант

14.IV.2005

(C) $2006 \mathrm{r}$.

ЯРЫКИН П. Н.*

\title{
УСТОЙЧИВОСТЬ НЕЛИНЕЙНОГО СТОХАСТИЧЕСКОГО ПРОЦЕССА, АППРОКСИМИРУЮЩЕГО СИСТЕМУ ВЗАИМОДЕЙСТВУЮЩИХ ЧАСТИЦ
}

\begin{abstract}
Рассматривается нелинейное стохастическое дифференциальное уравнение типа Маккина-Власова без «внешнего поля». Доказывается существование и сильная единственность решения уравнения. Далее доказывается существование и слабая единственность стационарного решения в классе распределений с заданным математическим ожиданием, а также сходимость по вероятности любого решения к стационарному распределению с соответствующим математическим ожиданием при больших временах.
\end{abstract}

Ключевые слова и фразы: нелинейный стохастический процесс, уравнение Маккина-Власова, устойчивость.

Введение Настоящая статья посвящена исследованию решения нелинейного стохастического дифференциального уравнения (СДУ) вида

$$
\tilde{X}_{t}=X_{0}+W_{t}+\int_{0}^{t} \int_{\mathbf{R}^{1}} b\left(\tilde{X}_{s}, y\right) \mu_{s}^{\tilde{X}}(d y) d s, \quad t \geqslant 0,
$$

где $\mu_{s}^{\tilde{X}}$ является распределением $\widetilde{X}_{s} \in \mathbf{R}^{1}, W_{t}$ - броуновское движение, не зависящее от начального значения $X_{0}, b\left(\tilde{X}_{s}, y\right)$ - функция попарного взаимодействия.

* Московский государственный университет им. М.В. Ломоносова, механикоматематический факультет, кафедра теории вероятностей, Ленинские горы, 119992 Москва, Россия; e-mail: yarykin@plushka.msk.ru 
Определим, что мы подразумеваем под решением нелинейного СДУ.

Пусть имеется (расширенная Р-нулевыми событиями) фильтрация $\mathbb{F}=\left(\mathscr{F}_{t}\right)_{t \geqslant 0}$, винеровский процесс $W=\left\{W_{t}, t \geqslant 0\right\}$ относительно $\mathbb{F}$ и начальное условие $\widetilde{X}_{0}=X_{0}$, измеримое относительно $\mathscr{F}_{0}$. Сильным решением нелинейного СДУ (1) на промежутке $[0, \infty)$ называется случайный процесс $\widetilde{X}=\left\{\tilde{X}_{t}, t \geqslant 0\right\}$, имеющий п.н. непрерывные траектории, согласованный с фильтрацией $\mathbb{F}$ и такой, что при подстановке его и семейства его распределений $\left\{\mu_{t}^{\tilde{X}}, t \geqslant 0\right\}$ в левую и правую части формулы (1) при каждом $t \geqslant 0$ получается равенство с вероятностью единица.

Это уравнение описывает случайный процесс, являющийся пределом среднего поля $(t-$ фиксировано, $N \rightarrow \infty)$ для выделенной частищы из системы $N$ взаимодействующих на расстоянии броуновских частиц, заданных системой СДУ

$$
X_{t}^{i, N}=X_{0}^{i}+W_{t}^{i}+\int_{0}^{t} \frac{1}{N} \sum_{j=1}^{N} b\left(X_{s}^{i, N}, X_{s}^{j, N}\right) d s, \quad t \geqslant 0 .
$$

В данной работе рассматривается симметричное взаимодействие, инвариантное относительно времени и сдвига:

$$
b(x, y)=-\beta(x-y)=-b(y, x),
$$

состоящее из двух компонент: линейно возрастающей силы притяжения и ограниченного липшицева возмушения

$$
\beta(x)=x+\beta_{1}(x)
$$

где

$$
\begin{array}{rrrr}
\exists A>0: & \left|\beta_{1}(x)\right| & \leqslant A, & x \in \mathbf{R}, \\
\exists \alpha>0: & \left|\beta_{1}(x)-\beta_{1}(y)\right| \leqslant \alpha|x-y|, & x, y \in \mathbf{R} .
\end{array}
$$

Пределом среднего поля называют утверждение о том, что на любом конечном интервале $[0, T] X_{t}^{i, N}$ стремится в среднем квадратичном при $N \rightarrow \infty$ к $\widetilde{X}_{t}$ (с тем же броуновским движением). Распространение хаоса - это ситуация, когда при любых $n, t$ распределения $X_{t}^{1, N}, \ldots, X_{t}^{n, N}$ асимптотически независимы при возрастании $N$.

Уравнения типа (1) неоднократно рассматривались ранее разными авторами. Так, одной из первых работ на эту тему была работа Г.П. Маккина [1], в которой для процессов, когда $\beta$ удовлетворяет условиям ограниченности, глобальной липшицевости и непрерывности, был получен предел среднего поля.

А.-С. Снитман в [5] получил предел среднего поля для глобально литшищева ограниченного $b(\cdot, \cdot)$, а также для случая локального взаимодействия.

Й. Тамура, в работах [3], [4], рассматривал процесс, близкий к (1), но при условии быстрого убывания на бесконечности функции взаимодействия $\beta$ и наличия сильного полиномиального «центростремительного» внешнего поля. В епо работах доказаны сушествование и единственность решения соответствуюшего стохастического уравнения, сушествование и единственность стационарного решения и сходимость по вариации любого решения к нему. Там же показано, что имеет место и распространение xaoca.

С. Бенашур, Б. Руанет, Д. Тале, П. Валуа в работах [6] и [7] получили результаты, аналогичные результатам Тамура, но уже для уравнения (1) (без дополнительного внешнего поля), в предположении не менее чем линейного роста функции $\beta$, ее локальной липшицевости, выпуклости и некоторых других технических условий.

В препринте [9] А.Ю. Веретенников рассматривает вопросы существования и единственности, предельного поведения нелинейного процесса при больших временах и распространения хаоса для симметричного взаимодействия, растущего при увеличении расстояния не более чем линейно (равномерно по пространству), при достаточно сильном внешнем поле, притягиваюшем процесс в 0 . Заметим, что непосредственное применение метода, использованного в [9], не позволяет получить результаты, изложенные в предлагаемой статье, в силу отсутствия внешнего поля. 
Эта работа является развитием работы автора [10], в которой рассматривался процесс (1) при $\beta(x)=x+\widehat{\alpha} \sin x$. В [10] были доказаны существование и единственность решения нелинейного СДУ при всех $\widehat{\alpha}$, сушествование и единственность стационарного решения при $\widehat{\alpha}<\widehat{\alpha}_{0}$ для некоторого $\widehat{\alpha}_{0}>\frac{1}{2}$ и слабая сходимость решения к стационарному (при малых $|\widehat{\alpha}|$ и некоторых дополнительных технических условиях). Также в работе [10] было показано, что при $\widehat{\alpha} \geqslant \widehat{\alpha}_{0}$ сушествует несколько стационарных решений.

В данной работе используются сушественно другие методы исследования предельного поведения процесса. В результате, ограничения на ядро взаимодействия стали сушественно слабее, для получения слабой сходимости перестала требоваться четность начального распределения, однако данный подход не позволил получить оценку скорости сходимости произвольного решения к стационарному.

1. Существование нелинейного стохастического процесса. Аналогично [10], докажем теорему о сушествовании и единственности решения уравнения (1). При этом, для удобства, обозначим $b_{1}(x, y):=-\beta_{1}(x-y)$. Тогда $b(x, y)=-(x-y)+$ $b_{1}(x, y)$.

Теорема 1. Уравнение (1) при начальном условии $X_{0}$ с конечньм первьим моментом имеет единственное сильное решение на промежутке $[0, \infty)$. Более того, математическое ожидание $\mathbf{E} \tilde{X}_{t}$ существует и ограничено.

Для доказательства потребуются следуюшие утверждения.

Утверждение 1 (следует из [2, гл. 1, теорема 3.3] при $V(x, t)=|x|+1$ ). Пусть $\widehat{b}(x, t)$ удовлетворяет условию Липшича, причем $\widehat{b}(0, t)$ интегрируема на конечных интервалах. Пусть тажже существует константа $c_{1}$ такая, что $\widehat{b}(x, t) \operatorname{sign}(x)<$ $c_{1}(1+|x|)$. Тогда сильное решение задачи

$$
X_{t}^{(\widehat{b})}=X_{0}+W_{t}+\int_{0}^{t} \widehat{b}\left(X_{s}^{(\widehat{b})}, s\right) d s
$$

существует и единственно при всех $t \geqslant 0$.

Утверждение 2 (следует из [2, гл. 1, доказательство теоремы 4.1]). В условивх утверждения 1 для любого $T>0$

$$
\sup _{0<t<T} \mathbf{E}\left|X_{t}^{(\widehat{b})}\right|<\infty .
$$

Д ок а за т ел ь с т о т е о ре мы 1. Для доказательства теоремы определим непрерывное сжимающее отображение на некотором замкнутом множестве в банаховом пространстве такое, что его неподвижная точка и только она будет соответствовать решению нелинейного уравнения.

1. Положим $\Lambda_{T}:=\{g: \mathbf{R} \times[0, T] \rightarrow \mathbf{R} \mid g(x, t)$ ограничена, $|g(x, t)-g(y, t)| \leqslant$ $\alpha|x-y|$ для всех $x, y \in \mathbf{R}$ и $t \in[0, T]\}$ (множество ограниченных и липшишевых по $x$ с константой $\alpha$ функций) и определим норму $\|g\|_{T}:=\sup _{x \in \mathbf{R}, t \in[0, T]}|g(x, t)|<\infty$.

Тогда множество $\Lambda_{T}$ является замкнутым подмножеством полного нормированного пространства.

2. Построим сжимающее отображение $\Gamma$ из $\Lambda_{T}$ в себя следующей цепочкой преобразований:

$$
g \rightarrow \widehat{b}^{(g)}(x, t):=g(x, t)-x+\mathbf{E} X_{0} \rightarrow Z_{t}^{(g)} \longrightarrow \Gamma g(x, t):=\mathbf{E} b\left(x, Z_{t}^{(g)}\right)+x-\mathbf{E} X_{0},
$$

где $Z_{t}^{(g)}$ является решением СДУ

$$
Z_{t}^{(g)}=X_{0}+W_{t}+\int_{0}^{t} \widehat{b}^{(g)}\left(Z_{s}^{(g)}, s\right) d s
$$

Легко увидеть, что все преобразования заданы корректно, поскольку для любого $g \in \Lambda_{T}$ функция $\widehat{b}^{(g)}(x, t)$ удовлетворяет всем условиям утверждения 1 и, следовательно, решение уравнения (4) сушествует и единственно. А корректность последнего перехода (сушествование $\left.\mathbf{E} b\left(x, Z_{t}^{(g)}\right)\right)$ следует из утверждения 2. 


\section{3. Докажем, что $\Gamma g \in \Lambda_{T}$.}

Случайный процесс $Z_{t}^{(g)}$ является сильным решением уравнения (4), и в силу неравенства (2) имеем ограниченность $\Gamma g$, а учитывая неравенство (3), имеем

$$
|\Gamma g(x, t)-\Gamma g(y, t)|=\left|\mathbf{E}\left(\beta_{1}\left(Z_{t}^{(g)}-x\right)-\beta_{1}\left(Z_{t}^{(g)}-y\right)\right)\right| \leqslant \alpha|x-y| .
$$

4. Легко доказать, что $\Gamma$ - непрерывное отображение относительно естественной метрики $\|g\|_{T}$.

Докажем, что $\Gamma-$ сжимающее отображение. Для любых $g, f \in \Lambda_{T}$, аналогично доказательству леммы 2.4 в [6], имеем

$$
Z_{t}^{(g)}-Z_{t}^{(f)}=\int_{0}^{t}\left(g\left(Z_{s}^{(g)}, s\right)-Z_{s}^{(g)}-f\left(Z_{s}^{(f)}, s\right)+Z_{s}^{(f)}\right) d s .
$$

Для всех $\gamma>1$ функция $x \rightarrow|x|^{\gamma}$ является $C^{1}$-функцией, следовательно, по формуле Ньютона-Лейбница и формуле производной сложной функции

$$
\begin{aligned}
\left|Z_{t}^{(g)}-Z_{t}^{(f)}\right|^{\gamma}= & \gamma \int_{0}^{t}\left|Z_{s}^{(g)}-Z_{s}^{(f)}\right|^{\gamma-1} \operatorname{sign}\left(Z_{s}^{(g)}-Z_{s}^{(f)}\right) \\
& \times\left(g\left(Z_{s}^{(g)}, s\right)-Z_{s}^{(g)}-f\left(Z_{s}^{(f)}, s\right)+Z_{s}^{(f)}\right) d s .
\end{aligned}
$$

Возьмем математическое ожидание от обеих частей. Поскольку $\left|Z_{t}^{(g)}-Z_{t}^{(f)}\right|^{\gamma} \geqslant 0$, то $\mathbf{E}\left|Z_{t}^{(g)}-Z_{t}^{(f)}\right|^{\gamma}$ определено (но может быть равно $+\infty$ ). Но тогда определено и математическое ожидание правой части.

Подынтегральное выражение (а следовательно, и весь интеграл) ограничено сверху некоторой константой. Действительно, $g$ и $f$ - ограниченные функции, следовательно, сушествует константа $A$ такая, что $|g(x, s)-f(y, s)| \leqslant A$. Значит, $\operatorname{sign}(x-y) \cdot(g(x, s)-f(y, s)) \leqslant A$ и $\operatorname{sign}(x-y) \cdot(-x+y)=-|x-y| \leqslant 0$. Обозначим $x=Z_{s}^{(g)}$ и $y=Z_{s}^{(f)}$. Тогда при $|x-y| \leqslant A$ подынтегральное выражение не превосходит $A^{\gamma}$, а при $|x-y|>A$ оно отрицательно. В совокупности получаем, что интеграл не превосходит $t A^{\gamma}$.

Но в этом случае мы вынуждены принять, что обе части равенства определены и конечны.

По теореме Фубини (она применима, поскольку подынтегральное выражение ограничено сверху некоторой константой $C$ ) мы можем внести знак математического ожидания под интеграл.

Перейдем к пределу при $\gamma \rightarrow 1+$. Модуль подынтегрального выражения ограничен при $1<\gamma \leqslant 2$ случайным процессом $\max \left\{C+1,\left|Z_{t}^{(g)}-Z_{t}^{(f)}\right|^{2}\right\}$. Поэтому по теореме о равномерной сходимости можно занести предел под интегралы. Неопределенность типа $0^{0}$, возникаюшая в подынтегральном выражении при $Z_{t}^{(g)}=Z_{t}^{(f)}$, не мешает переходу к пределу в правой части, поскольку в этом случае $\operatorname{sign}\left(Z_{t}^{(g)}-Z_{t}^{(f)}\right)=0$ и $\operatorname{sign}\left(Z_{t}^{(g)}-Z_{t}^{(f)}\right) \cdot\left|Z_{t}^{(g)}-Z_{t}^{(f)}\right|^{0}=0$. Получаем:

$$
\begin{aligned}
\mathbf{E} \mid Z_{s}^{(g)} & -Z_{s}^{(f)} \mid=\int_{0}^{t} \mathbf{E} \operatorname{sign}\left(Z_{s}^{(g)}-Z_{s}^{(f)}\right)\left(g\left(s, Z_{s}^{(g)}\right)-f\left(s, Z_{s}^{(f)}\right)-Z_{s}^{(g)}+Z_{s}^{(f)}\right) d s \\
= & -\int_{0}^{t} \mathbf{E} \operatorname{sign}\left(Z_{t}^{(g)}-Z_{t}^{(f)}\right)\left(Z_{t}^{(g)}-Z_{t}^{(f)}\right) d s \\
& +\int_{0}^{t} \mathbf{E} \operatorname{sign}\left(Z_{s}^{(g)}-Z_{s}^{(f)}\right)\left(\left(g\left(s, Z_{s}^{(g)}\right)-g\left(s, Z_{s}^{(f)}\right)\right)+\left(g\left(s, Z_{s}^{(f)}\right)-f\left(s, Z_{s}^{(f)}\right)\right)\right) d s \\
= & -\int_{0}^{t} \mathbf{E}\left|Z_{s}^{(g)}-Z_{s}^{(f)}\right| d s+\int_{0}^{t} \mathbf{E} \operatorname{sign}\left(Z_{s}^{(g)}-Z_{s}^{(f)}\right)\left(g\left(s, Z_{s}^{(g)}\right)-g\left(s, Z_{s}^{(f)}\right)\right) d s \\
& +\int_{0}^{t} \mathbf{E} \operatorname{sign}\left(Z_{s}^{(g)}-Z_{s}^{(f)}\right)\left(g\left(s, Z_{s}^{(f)}\right)-f\left(s, Z_{s}^{(f)}\right)\right) d s \\
\leqslant & (\alpha-1) \int_{0}^{t} \mathbf{E}\left|Z_{s}^{(g)}-Z_{s}^{(f)}\right| d s+t\|g-f\|_{T},
\end{aligned}
$$


следовательно, по лемме Гронуолла

$$
\mathbf{E}\left|Z_{t}^{(g)}-Z_{t}^{(f)}\right| \leqslant \frac{t}{2}\|g-f\|_{T} e^{(\alpha-1) t / 2} \leqslant \frac{T}{2}\|g-f\|_{T} e^{(\alpha-1) T / 2},
$$

но $|\Gamma g(y, t)-\Gamma f(y, t)|=\left|\mathbf{E}\left(\beta_{1}\left(Z_{t}^{(g)}-y\right)-\beta_{1}\left(Z_{t}^{(f)}-y\right)\right)\right| \leqslant \alpha \mathbf{E}\left|Z_{t}^{(g)}-Z_{t}^{(f)}\right|$, поэтому

$$
\|\Gamma g(y, t)-\Gamma f(y, t)\|_{T} \leqslant \alpha \frac{T}{2}\|g-f\|_{T} e^{\alpha T / 2} .
$$

Следовательно, при $T=1 / \alpha$ отображение $\Gamma$ является сжимающим.

5. По теореме о сжимаюшем отображении мы получаем существование и единственность на отрезке $[0, T]$ сильного решения $X_{t}$ уравнения (1).

Точнее, по теореме о сжимающем отображении мы получаем существование и единственность неподвижной точки $g \in \Lambda_{T}$ отображения $\Gamma$. Но точка $g$ является неподвижной относительно отображения $\Gamma$ тогда и только тогда, когда $Z_{t}^{(g)}$ является решением СДУ (1).

6. Поскольку оценка коэффициента сжатия зависит только от $\alpha$ и $T$, то, рассматривая уравнение $(1)$ на отрезке $[k T,(k+1) T]$ с начальным условием $X_{k T}$, равным конечному значению $X_{t}$ на отрезке $[(k-1) T, k T]$, мы получим существование и единственность сильного решения уравнения (1) на отрезке $[k T,(k+1) T]$. Следовательно, теорема доказана.

Лемма 1. Пусть $\widetilde{X}_{t}$ - сильное решение уравнения (1). Тогда $\mathbf{E} \widetilde{X}_{t} \equiv$ const.

Д ок а з а те л ь т в о. Сушествование рассматриваемого математического ожидания доказано в теореме 1 .

Возьмем математическое ожидание от обеих частей уравнения (1). Получим

$$
\mathbf{E} \tilde{X}_{t}=\mathbf{E} X_{0}+\int_{0}^{t} \iint b(x, y) \mu_{s}^{\tilde{X}}(d y) \mu_{s}^{\tilde{X}}(d x) d s .
$$

Но в силу кососимметричности функции $b$ подынтегральное выражение тождественно нулевое.

3 а м е ч а н и е 1 . Без ограничения общности далее везде будем считать, что математическое ожидание $\widetilde{X}_{t}$ существует и равно 0 .

Действительно, мы можем рассмотреть процесс $\widetilde{Z}_{t}=\widetilde{X}_{t}-\mathbf{E} X_{0}$, применить к нему последуюшие теоремы и затем сделать обратный переход.

2. Предельное поведение процессов. Пусть даны $X_{0}^{1}, \ldots, X_{0}^{N}, \ldots$ - независимые одинаково распределенные случайные величины и $W_{t}^{1}, \ldots, W_{t}^{N}, \ldots$ - независимые стандартные броуновские движения, причем вся эта система случайных величин и броуновских движений независима в совокупности.

Рассмотрим систему из $N$ попарно взаимодействующих идентичных броуновских частиц:

$$
d X_{t}^{i, N}=d W_{t}^{i}+\frac{1}{N} \sum_{j=1}^{N} b\left(X_{t}^{i, N}, X_{t}^{j, N}\right) d t, \quad t \geqslant 0,
$$

с начальным условием $\left(X_{0}^{1}, \ldots, X_{0}^{N}\right)$.

Также рассмотрим решения нелинейных СДУ

$$
d \widetilde{X}_{t}^{i}=d W_{t}^{i}+\left(\int_{\mathbf{R}^{1}} b\left(\widetilde{X}_{t}^{i}, y\right) \mu_{t}^{\widetilde{X}}(d y)\right) d t, \quad t \geqslant 0,
$$

с теми же начальными условиями, где $\mu_{t}^{\tilde{X}}$ (строго говоря, $\mu_{t}^{\tilde{X}^{i}}$ ) является законом распределения (мерой) случайной величины $\widetilde{X}_{t}^{i}$. В указанных условиях все $\widetilde{X}_{t}^{i}$ независимы и одинаково распределены.

Рассмотрим вспомогательные случайные процессы

$$
Y_{t}^{i, N}:=X_{t}^{i, N}-\frac{1}{N} \sum_{j=1}^{N} X_{t}^{j, N}, \quad \tilde{Y}_{t}^{i, N}:=\tilde{X}_{t}^{i}-\frac{1}{N} \sum_{j=1}^{N} \tilde{X}_{t}^{j} .
$$


Лемма 2. Пусть $\mathrm{E} X_{0}=0 u \mathrm{E} X_{0}^{2}$ конечно. Тогда при $\alpha<\frac{1}{4}$ существует константа $C$ такал, что

$$
\sup _{t} \mathrm{E}\left|Y_{t}^{i, N}-\tilde{Y}_{t}^{i, N}\right|^{2} \leqslant \frac{C}{N}
$$

Д о к а з а т е л ь с т в о. Для любой серии случайных величин $Z_{t}^{1[, N]}, \ldots$, $Z_{t}^{N[, N]}$ с одинаковым распределением обозначим:

(i) $\widehat{\mu}_{t}^{N, Z}$ - выборочное (эмпирическое) распределение $Z_{t}^{1[, N]}, \ldots, Z_{t}^{N[, N]}$ (т.е. $\widehat{\mu}_{t}^{N, Z}(A)=N^{-1} \sum I\left\{Z_{t}^{i[, N]} \in A\right\}$ для любого множества $\left.A \subset \mathbf{R}^{1}\right)$;

(ii) $\mu_{t}^{[N,] Z}$ - распределение $Z_{t}^{i[, N]}$.

Кроме того, для любой случайной величины $Z$ и любой вероятностной меры $\nu$ положим $b[Z, \nu]:=\int b(Z, y) \nu(d y), b_{1}[Z, \nu]:=\int b_{1}(Z, y) \nu(d y)$.

Тогда в силу формул (5)-(7) и линейности дифференциалов новые процессы будут удовлетворять следуюшей системе уравнений

$$
\begin{aligned}
& d Y_{t}^{i, N}=d W_{t}^{i}-\left(\frac{1}{N}, \ldots, \frac{1}{N}\right) \cdot d \vec{W}_{t}+b\left[X_{t}^{i, N}, \widehat{\mu}_{t}^{N, X}\right] d t-\frac{1}{N} \sum_{j=1}^{N} b\left[X_{t}^{j, N}, \widehat{\mu}_{t}^{N, X}\right] d t, \\
& d \widetilde{Y}_{t}^{i, N}=d W_{t}^{i}-\left(\frac{1}{N}, \ldots, \frac{1}{N}\right) \cdot d \vec{W}_{t}+b\left[\widetilde{X}_{t}^{i}, \mu_{t}^{\widetilde{X}}\right] d t-\frac{1}{N} \sum_{j=1}^{N} b\left[\tilde{X}_{t}^{j}, \mu_{t}^{\widetilde{X}}\right] d t .
\end{aligned}
$$

В силу трансляционной инвариантности взаимодействия

$$
\begin{aligned}
b\left[X_{t}^{i, N}, \widehat{\mu}_{t}^{N, X}\right] & =\frac{1}{N} \sum_{k=1}^{N} b\left(X_{t}^{i, N}, X_{t}^{k, N}\right)=-\frac{1}{N} \sum_{k=1}^{N} \beta\left(X_{t}^{i, N}-X_{t}^{k, N}\right) \\
& =-\frac{1}{N} \sum_{k=1}^{N} \beta\left(X_{t}^{i, N}-\frac{1}{N} \sum_{j=1}^{N} X_{t}^{j, N}+\frac{1}{N} \sum_{j=1}^{N} X_{t}^{j, N}-X_{t}^{k, N}\right) \\
& =-\frac{1}{N} \sum_{k=1}^{N} \beta\left(Y_{t}^{i, N}-Y_{t}^{k, N}\right)=\frac{1}{N} \sum_{k=1}^{N} b\left(Y_{t}^{i, N}, Y_{t}^{k, N}\right)=b\left[Y_{t}^{i, N}, \widehat{\mu}_{t}^{N, Y}\right]
\end{aligned}
$$

Значит, уравнение (8) можно переписать в виде

$$
d Y_{t}^{i, N}=d W_{t}^{i}-\left(\frac{1}{N}, \ldots, \frac{1}{N}\right) \cdot d \vec{W}_{t}+b\left[Y_{t}^{i, N}, \widehat{\mu}_{t}^{N, Y}\right] d t-\frac{1}{N} \sum_{j=1}^{N} b\left[Y_{t}^{j, N}, \widehat{\mu}_{t}^{N, Y}\right] d t .
$$

Обратим внимание, что трансляционная инвариантность взаимодействия не позволяет провести подобную замену координат для $b\left[\widetilde{X}_{t}^{i}, \mu_{t}^{\widetilde{X}}\right]$.

Рассмотрим дифференциал разности нелинейного (уравнение (9)) и многочастичного (линейного, уравнение (10)) случайных процессов:

$$
\begin{aligned}
& d\left(Y_{t}^{i, N}-\tilde{Y}_{t}^{i, N}\right) \\
& =\left(b\left[Y_{t}^{i, N}, \widehat{\mu}_{t}^{N, Y}\right]-\frac{1}{N} \sum_{j=1}^{N} b\left[Y_{t}^{j, N}, \widehat{\mu}_{t}^{N, Y}\right]-b\left[\tilde{X}_{t}^{i}, \mu_{t}^{\tilde{X}}\right]+\frac{1}{N} \sum_{j=1}^{N} b\left[\tilde{X}_{t}^{j}, \mu_{t}^{\tilde{X}}\right]\right) d t \\
& =\left(b_{1}\left[Y_{t}^{i, N}, \widehat{\mu}_{t}^{N, Y}\right]-\frac{1}{N} \sum_{j=1}^{N} b_{1}\left[Y_{t}^{j, N}, \widehat{\mu}_{t}^{N, Y}\right]-b_{1}\left[\tilde{X}_{t}^{i}, \mu_{t}^{\widetilde{X}}\right]+\frac{1}{N} \sum_{j=1}^{N} b_{1}\left[\tilde{X}_{t}^{i}, \mu_{t}^{\tilde{X}}\right]\right) d t \\
& \quad+\left(\left(-Y_{t}^{i, N}+\frac{1}{N} \sum_{k=1}^{N} Y_{t}^{k, N}\right)-\frac{1}{N} \sum_{j=1}^{N}\left(-Y_{t}^{j, N} * \frac{1}{N} \sum_{k=1}^{N} Y_{t}^{k, N}\right)\right. \\
& \left.\quad-\left(-\widetilde{X}_{t}^{i}\right)+\frac{1}{N} \sum_{j=1}^{N}\left(-\widetilde{X}_{t}^{j}\right)\right) d t .
\end{aligned}
$$

В последнем равенстве было использовано, что $\mathbf{E} \tilde{X}_{t}^{j}=0$. 
Учитывая, что $\sum_{j=1}^{N} Y_{t}^{j, N} \equiv 0$, получаем

$$
\begin{aligned}
& d\left(Y_{t}^{i, N}-\tilde{Y}_{t}^{i, N}\right)=(\left(-Y_{t}^{i, N}+0\right)-\underbrace{\frac{1}{N} \sum_{j=1}^{N}\left(-Y_{t}^{j, N}+0\right)}_{0}+\underbrace{\left(\tilde{X}_{t}^{i}-\frac{1}{N} \sum_{j=1}^{N} \tilde{X}_{t}^{j}\right)}_{\widetilde{Y}_{t}^{i, N}}) d t \\
& \quad+\left(b_{1}\left[Y_{t}^{i, N}, \widehat{\mu}_{t}^{N, Y}\right]-\frac{1}{N} \sum_{j=1}^{N} b_{1}\left[Y_{t}^{j, N}, \widehat{\mu}_{t}^{N, Y}\right]-b_{1}\left[\tilde{X}_{t}^{i}, \mu_{t}^{\tilde{X}}\right]+\frac{1}{N} \sum_{j=1}^{N} b_{1}\left[\tilde{X}_{t}^{i}, \mu_{t}^{\widetilde{X}}\right]\right) d t \\
& =-\left(Y_{t}^{i, N}-\tilde{Y}_{t}^{i, N}\right) d t \\
& \quad+\left(b_{1}\left[Y_{t}^{i, N}, \widehat{\mu}_{t}^{N, Y}\right]-\frac{1}{N} \sum_{j=1}^{N} b_{1}\left[Y_{t}^{j, N}, \widehat{\mu}_{t}^{N, Y}\right]-b_{1}\left[\tilde{X}_{t}^{i}, \mu_{t}^{\widetilde{X}}\right]+\frac{1}{N} \sum_{j=1}^{N} b_{1}\left[\tilde{X}_{t}^{i}, \mu_{t}^{\tilde{X}_{t}}\right]\right) d t .
\end{aligned}
$$

Согласно формуле Ито,

$$
\begin{aligned}
\frac{d}{d t} \mathbf{E}\left(Y_{t}^{i, N}-\tilde{Y}_{t}^{i, N}\right)^{2}= & -2 \mathbf{E}\left|Y_{t}^{i, N}-\tilde{Y}_{t}^{i, N}\right|^{2} \\
& +2 \mathbf{E}\left(Y_{t}^{i, N}-\tilde{Y}_{t}^{i, N}\right)\left(b_{1}\left[Y_{t}^{i, N}, \widehat{\mu}_{t}^{N, Y}\right]-b_{1}\left[\tilde{X}_{t}^{i}, \mu_{t}^{\widetilde{X}}\right]\right) \\
& -\frac{2}{N} \mathbf{E} \sum_{j=1}^{N}\left(Y_{t}^{i, N}-\tilde{Y}_{t}^{i, N}\right)\left(b_{1}\left[Y_{t}^{j, N}, \widehat{\mu}_{t}^{N, Y}\right]-b_{1}\left[\tilde{X}_{t}^{j}, \mu_{t}^{\tilde{X}}\right]\right) \\
= & -2 \mathbf{E}\left|Y_{t}^{i, N}-\tilde{Y}_{t}^{i, N}\right|^{2}+2 A^{i, i}-\frac{2}{N} \sum_{j=1}^{N} A^{i, j},
\end{aligned}
$$

где $A^{i, j}:=\mathbf{E}\left(Y_{t}^{i, N}-\tilde{Y}_{t}^{i, N}\right)\left(b_{1}\left[Y_{t}^{j, N}, \widehat{\mu}_{t}^{N, Y}\right]-b_{1}\left[\tilde{X}_{t}^{j}, \mu_{t}^{\tilde{X}}\right]\right)=\mathbf{E}\left(Y_{t}^{i, N}-\tilde{Y}_{t}^{i, N}\right)\left(\left(b_{1}\left[Y_{t}^{j, N}, \widehat{\mu}_{t}^{N, Y}\right]-\right.\right.$ $\left.\left.b_{1}\left[\widetilde{Y}_{t}^{j, N}, \widehat{\mu}_{t}^{N, \widetilde{Y}}\right]\right)+\left(b_{1}\left[\tilde{Y}_{t}^{j, N}, \widehat{\mu}_{t}^{N, \tilde{Y}}\right]-b_{1}\left[\tilde{X}_{t}^{j}, \mu_{t}^{\tilde{X}}\right]\right)\right)$. Раскрывая скобки, получим два слагаемых и во втором множителе второго слагаемого, опять используя трансляционную инвариантность, заменим $\widetilde{Y}$ на $\widetilde{X}$ :

$$
\begin{aligned}
A^{i, j}= & \mathbf{E}\left(Y_{t}^{i, N}-\widetilde{Y}_{t}^{i, N}\right)\left(b_{1}\left[Y_{t}^{j, N}, \widehat{\mu}_{t}^{N, Y}\right]-b_{1}\left[\tilde{Y}_{t}^{j, N}, \widehat{\mu}_{t}^{N, \widetilde{Y}}\right]\right) \\
& +\mathbf{E}\left(Y_{t}^{i, N}-\tilde{Y}_{t}^{i, N}\right)\left(b_{1}\left[\tilde{X}_{t}^{j}, \widehat{\mu}_{t}^{\tilde{X}}\right]-b_{1}\left[\tilde{X}_{t}^{j}, \mu_{t}^{\tilde{X}}\right]\right)=: A_{1}^{i, j}+A_{2}^{i, j} .
\end{aligned}
$$

Таким образом, получаем, что первое слагаемое, $A_{1}^{i, j}$, содержит только выборочные меры, а второе, $A_{2}^{i, j}$, - только величины нелинейного процесса.

Оценим $A_{1}^{i, j}$ и $A_{2}^{i, j}$ отдельно.

Начнем с $A_{1}^{i, j}$. Для этого прежде всего заменим выборочные меры и функцию $b(x, y)$ на суммы:

$$
\begin{aligned}
A_{1}^{i, j} & =\frac{1}{N} \sum_{k=1}^{N} \mathbf{E}\left(Y_{t}^{i, N}-\tilde{Y}_{t}^{i, N}\right)\left(b_{1}\left(Y_{t}^{j, N}, Y_{t}^{k, N}\right)-b_{1}\left(\tilde{Y}_{t}^{j, N}, \tilde{Y}_{t}^{k, N}\right)\right) \\
& =\frac{1}{N} \sum_{k=1}^{N} \mathbf{E}\left(Y_{t}^{i, N}-\tilde{Y}_{t}^{i, N}\right)\left(-\beta_{1}\left(Y_{t}^{j, N}-Y_{t}^{k, N}\right)+\beta_{1}\left(\tilde{Y}_{t}^{j, N}-\tilde{Y}_{t}^{k, N}\right)\right) .
\end{aligned}
$$

Учитывая (3) и перегруппировывая, получаем

$$
\begin{aligned}
\left|A_{1}^{i, j}\right| & \leqslant \frac{\alpha}{N} \sum_{k=1}^{N} \mathbf{E}\left|\left(Y_{t}^{i, N}-\tilde{Y}_{t}^{i, N}\right)\left(\left(Y_{t}^{j, N}-Y_{t}^{k, N}\right)-\left(\tilde{Y}_{t}^{j, N}-\tilde{Y}_{t}^{k, N}\right)\right)\right| \\
& =\frac{\alpha}{N} \sum_{k=1}^{N} \mathbf{E}\left|\left(Y_{t}^{i, N}-\tilde{Y}_{t}^{i, N}\right)\left(\left(Y_{t}^{j, N}-\tilde{Y}_{t}^{j, N}\right)-\left(Y_{t}^{k, N}-\tilde{Y}_{t}^{k, N}\right)\right)\right| .
\end{aligned}
$$


Следовательно,

$$
\begin{aligned}
\left|A_{1}^{i, j}\right| \leqslant & \alpha \mathbf{E}\left|\left(Y_{t}^{i, N}-\tilde{Y}_{t}^{i, N}\right)\left(Y_{t}^{j, N}-\tilde{Y}_{t}^{j, N}\right)\right|+\frac{\alpha}{N} \sum_{k=1}^{N} \mathbf{E}\left|\left(Y_{t}^{i, N}-\tilde{Y}_{t}^{i, N}\right)\left(Y_{t}^{k, N}-\tilde{Y}_{t}^{k, N}\right)\right| \\
\leqslant & \alpha\left(\mathbf{E}\left(Y_{t}^{i, N}-\tilde{Y}_{t}^{i, N}\right)^{2}\right)^{1 / 2}\left(\mathbf{E}\left(Y_{t}^{j, N}-\tilde{Y}_{t}^{j, N}\right)^{2}\right)^{1 / 2} \\
& +\frac{\alpha}{N} \sum_{k=1}^{N}\left(\mathbf{E}\left(Y_{t}^{i, N}-\tilde{Y}_{t}^{i, N}\right)^{2}\right)^{1 / 2}\left(\mathbf{E}\left(Y_{t}^{k, N}-\tilde{Y}_{t}^{k, N}\right)^{2}\right)^{1 / 2}
\end{aligned}
$$

и, учитывая симметричность многомерного распределения, получаем

$$
\left|A_{1}^{i, j}\right| \leqslant 2 \alpha \mathrm{E}\left(Y_{t}^{i, N}-\tilde{Y}_{t}^{i, N}\right)^{2} .
$$

Рассмотрим далее $A_{2}^{i, j}$. Для этого также заменим выборочные меры на суммы:

$$
\begin{aligned}
\left|A_{2}^{i, j}\right| & \leqslant \mathbf{E}\left|\left(Y_{t}^{i, N}-\tilde{Y}_{t}^{i, N}\right)\left(b_{1}\left[\tilde{X}_{t}^{j}, \widehat{\mu}_{t}^{\tilde{X}}\right]-b_{1}\left[\tilde{X}_{t}^{j}, \mu_{t}^{\tilde{X}}\right]\right)\right| \\
& =\mathbf{E}\left|\frac{1}{N} \sum_{k=1}^{N}\left(Y_{t}^{i, N}-\tilde{Y}_{t}^{i, N}\right)\left(b_{1}\left(\tilde{X}_{t}^{j}, \tilde{X}_{t}^{k}\right)-b_{1}\left[\tilde{X}_{t}^{j}, \mu_{t}^{\tilde{X}}\right]\right)\right| \\
& \leqslant \frac{1}{N}\left(\mathbf{E}\left(Y_{t}^{i, N}-\tilde{Y}_{t}^{i, N}\right)^{2}\right)^{1 / 2}\left(\mathbf{E}\left(\sum_{k=1}^{N}\left(b_{1}\left(\tilde{X}_{t}^{j}, \tilde{X}_{t}^{k}\right)-b_{1}\left[\tilde{X}_{t}^{j}, \mu_{t}^{\widetilde{X}}\right]\right)\right)^{2}\right)^{1 / 2} .
\end{aligned}
$$

Теперь заметим (аналогично [5]), что случайные величины $\left(b_{1}\left(\widetilde{X}_{t}^{j}, \widetilde{X}_{t}^{k}\right)-b_{1}\left[\widetilde{X}_{t}^{j}, \mu_{t}^{\widetilde{X}}\right]\right)$ и $\left(b_{1}\left(\tilde{X}_{t}^{j}, \tilde{X}_{t}^{l}\right)-b_{1}\left[\tilde{X}_{t}^{j}, \mu_{t}^{\tilde{X}}\right]\right)$ некоррелированы при $k \neq l$ : произведение их математических ожиданий равно 0 , так как при $j \neq k$

$$
\mathbf{E} b_{1}\left(\tilde{X}_{t}^{j}, \tilde{X}_{t}^{k}\right)=\mathbf{E}\left(\mathbf{E}\left(b_{1}\left(\tilde{X}_{t}^{j}, \tilde{X}_{t}^{k}\right) \mid \tilde{X}_{t}^{j}\right)\right)=\mathbf{E}\left(\int_{\mathbf{R}^{1}} b_{1}\left(\tilde{X}_{t}^{j}, y\right) \mu(d y)\right)=\mathbf{E} b_{1}\left[\tilde{X}_{t}^{j}, \mu_{t}^{\widetilde{X}}\right]
$$

и имеем

$$
\begin{aligned}
& \mathbf{E}\left(b_{1}\left(\tilde{X}_{t}^{j}, \tilde{X}_{t}^{k}\right)-b_{1}\left[\tilde{X}_{t}^{j}, \mu_{t}^{\tilde{X}}\right]\right)\left(b_{1}\left(\tilde{X}_{t}^{j}, \tilde{X}_{t}^{l}\right)-b_{1}\left[\tilde{X}_{t}^{j}, \mu_{t}^{\widetilde{X}}\right]\right) \\
& =\mathbf{E}\left(\mathbf{E}\left(\left(b_{1}\left(\tilde{X}_{t}^{j}, \tilde{X}_{t}^{k}\right)-b_{1}\left[\tilde{X}_{t}^{j}, \mu_{t}^{\tilde{X}}\right]\right)\left(b_{1}\left(\tilde{X}_{t}^{j}, \widetilde{X}_{t}^{l}\right)-b_{1}\left[\tilde{X}_{t}^{j}, \mu_{t}^{\widetilde{X}}\right]\right) \mid \tilde{X}_{t}^{j}\right)\right) \\
& =\mathbf{E}\left(\mathbf{E}\left(b_{1}\left(\tilde{X}_{t}^{j}, \tilde{X}_{t}^{k}\right)-b_{1}\left[\tilde{X}_{t}^{j}, \mu_{t}^{\tilde{X}}\right] \mid \tilde{X}_{t}^{j}\right) \mathbf{E}\left(b_{1}\left(\tilde{X}_{t}^{j}, \tilde{X}_{t}^{l}\right)-b_{1}\left[\tilde{X}_{t}^{j}, \mu_{t}^{\widetilde{X}}\right] \mid \tilde{X}_{t}^{j}\right)\right),
\end{aligned}
$$

но при $j \neq k$ первый (а иначе второй, поскольку тогда $j \neq l$ ) множитель в правой части обрашается в 0 .

Кроме того, $\left(b_{1}\left(\tilde{X}_{t}^{j}, \tilde{X}_{t}^{k}\right)-b_{1}\left[\tilde{X}_{t}^{j}, \mu_{t}^{\widetilde{X}}\right]\right)$ имеют конечный второй момент, не превосходящий $4 A^{2}$. Таким образом, получаем

$$
\begin{aligned}
\left|A_{2}^{i, j}\right| & \leqslant \frac{1}{N}\left(\mathbf{E}\left(Y_{t}^{i, N}-\tilde{Y}_{t}^{i, N}\right)^{2}\right)^{1 / 2}\left(\sum_{k=1}^{N} \mathbf{E}\left(b_{1}\left(\tilde{X}_{t}^{j}, \tilde{X}_{t}^{k}\right)-b_{1}\left[\tilde{X}_{t}^{j}, \mu_{t}^{\tilde{X}}\right]\right)^{2}\right)^{1 / 2} \\
& \leqslant \frac{8 A^{2}}{N^{1 / 2}}\left(\mathbf{E}\left(Y_{t}^{i, N}-\tilde{Y}_{t}^{i, N}\right)^{2}\right)^{1 / 2}
\end{aligned}
$$

Следовательно, полагая $\gamma(t):=\mathbf{E}\left|Y_{t}^{i, N}-\tilde{Y}_{t}^{i, N}\right|^{2}$, можем записать

$$
\frac{\gamma^{\prime}(t)}{2} \leqslant-(1-4 \alpha) \gamma(t)+\frac{8 A^{2}}{N^{1 / 2}} \gamma^{1 / 2}(t) .
$$

Заметим, что стационарное решение уравнения, соответствующего неравенству $(11), \gamma_{0}(t)=\gamma_{0}=64 A^{4} /\left((1-4 \alpha)^{2} N\right)$, лежит в зоне действия теоремы о существовании и единственности решения задачи Коши. Следовательно, $\gamma(t)$ не сможет пересечь уровень $\gamma_{0}$. Поэтому, учитывая, что $\gamma(0)=0<\gamma_{0}$, получаем, что

что доказывает лемму.

$$
\gamma(t) \leqslant \frac{64 A^{4}}{(1-4 \alpha)^{2} N},
$$


Теорема 2. В условиях леммы 2 (т.е. если $\mathbf{E} X_{0}=0$, $\mathbf{E} X_{0}^{2}$ конечно $u \alpha<\frac{1}{4}$ ) получаем, что имеет место слабая сходимость $\widetilde{X}_{t} \Rightarrow \widetilde{X}_{\infty}$ nрu $t \rightarrow \infty$.

Д о к а з а т е л ь с т в. При доказательстве мы используем равномерную ограниченность дисперсии $\widetilde{X}_{t}^{j}$. Докажем ее. Из уравнения (6) по формуле Ито получаем, что $d\left(\widetilde{X}_{t}^{i}\right)^{2}=2 \widetilde{X}_{t}^{i} d W_{t}^{i}+2 \widetilde{X}_{t}^{i} b\left[\widetilde{X}_{t}^{i}, \mu_{t}^{\widetilde{X}}\right] d t+d t$. Следовательно,

$$
\begin{aligned}
\frac{d}{d t} \mathbf{E}\left(\tilde{X}_{t}^{i}\right)^{2} & =2 \mathbf{E} \tilde{X}_{t}^{i} b\left[\tilde{X}_{t}^{i}, \mu_{t}^{\tilde{X}}\right]+1=-2 \mathbf{E}\left(\tilde{X}_{t}^{i}\right)^{2}+2 \mathbf{E} \tilde{X}_{t}^{i} b_{1}\left[\tilde{X}_{t}^{i}, \mu_{t}^{\tilde{X}}\right]+1 \\
& \leqslant-2 \mathbf{E}\left(\tilde{X}_{t}^{i}\right)^{2}+2 \alpha\left(\mathbf{E}\left(\tilde{X}_{t}^{i}\right)^{2}\right)^{1 / 2}+1 .
\end{aligned}
$$

Решив соответствуюшее дифференциальное уравнение относительно $\left(\mathbf{E}\left(\tilde{X}_{t}^{i}\right)^{2}\right)^{1 / 2}$ и найдя его стационарное решение $\left(\alpha+\sqrt{\alpha^{2}+2}\right) / 2=: c_{1}$, заметим, что при $\mathbf{E}\left(\widetilde{X}_{t}^{i}\right)^{2}>c_{1}^{2}$ правая часть (12) отрицательна, следовательно,

$$
\mathbf{E}\left(\tilde{X}_{t}^{i}\right)^{2} \leqslant \max \left\{\mathbf{E}\left(\tilde{X}_{0}^{i}\right)^{2}, c_{1}^{2}\right\}<\infty .
$$

Покажем, что при неограниченном возрастании $N$ имеет место равномерная по времени сходимость $Y_{t}^{i, N}$ к $\widetilde{X}_{t}^{i}$ в среднем квадратичном.

В силу равномерной ограниченности дисперсии и независимости $\widetilde{X}_{t}^{j}$ имеем $\sup _{t \geqslant 0} \mathbf{E}\left(\tilde{Y}_{t}^{i, N}-\tilde{X}_{t}^{i}\right)^{2}=N^{-2} \sup _{t \geqslant 0} \sum_{j=1}^{N} \mathbf{E}\left(\tilde{X}_{t}^{j}\right)^{2} \leqslant C_{1} / N$

Следовательно,

$$
\sup _{t \geqslant 0} \mathrm{E}\left|Y_{t}^{i, N}-\widetilde{X}_{t}^{i}\right|^{2} \leqslant \frac{C_{2}}{N} .
$$

Далее, заметим, что в силу [8, теорема 5] уравнения (10) задают эргодический марковский процесс (условия теоремы выполняются для любого $r$ и $m \geqslant 2$ ). Следовательно, существует единственная инвариантная мера $\mu^{N, Y}$, причем $\| \mu_{t}^{N, Y}-$ $\mu^{N, Y} \|_{T V} \rightarrow 0$ при $t \rightarrow \infty$.

По теореме о двойном пределе получаем существование двойного и равных ему повторных пределов $\mu_{t}^{N, Y}$, в частности, существует $\mu_{\infty}^{\widetilde{X}}:=\lim _{N \rightarrow \infty} \mu^{N, Y}$ и $\mu_{t}^{\widetilde{X}} \rightarrow \mu_{\infty}^{\widetilde{X}}$ при $t \rightarrow \infty$. Теорема доказана.

Автор выражает благодарность научному руководителю А.Д. Маните за постоянное внимание к работе и ценные советы и М. А. Урусову за подробное обсуждение теоремы о сушествовании решения.

\section{СПИСОК ЛИТЕРАТУРЫ}

1. McKean H.P., Jr. A class of Markov processes associated with nonlinear parabolic equations. - Proc. Natl. Acad. Sci. USA, 1966, v. 56, p. 1907-1911.

2. Хасьминский Р. З. Устойчивость систем дифференциальных уравнений при случайных возмущениях их параметров. М.: Наука, 1969, 367 с.

3. Tamura $Y$. On asymptotic behaviors of the solution of a nonlinear diffusion equation. - J. Fac. Sci. Univ. Tokyo, 1984, v. 31, № 1, p. 195-221.

4. Tamura $Y$. Free energy and the convergence of distributions of diffusion processes of McKean type. - J. Fac. Sci. Univ. Tokyo, 1987, v. 34, № 2, p. 443-484.

5. Sznitman A.-S. Topics in propagation of chaos. - Lecture Notes in Math., 1991, v. 1464, p. $165-251$.

6. Benachour S., Roynette B., Talay D., Vallois P. Nonlinear self-stabilizing processes. I. Existence, invariant probability, propagation of chaos. - Stochastic Process. Appl., 1998 , v. 75 , № 2 , p. 173-201.

7. Benachour S., Roynette B., Vallois $P$. Nonlinear self-stabilizing processes. II. Convergence to invariant probability. - Stochastic Process. Appl., 1998, v. 75, № 2, p. 203224.

8. Веретенников $A$. Ю. О полиномиальном перемешивании и скорости сходимости для стохастических дифференциальных и разностных уравнений. - Теория вероятн. и ее примен., 1999, т. 44 , в. 2 , с. $312-327$. 
9. Veretennikov A. Yu. On ergodic measures for McKean-Vlasov stochastic equations. Preprint NI03066. Cambridge: Isaac Newton Inst. for Math. Sci., 2003, 13 p. (http://www.newton.cam.ac.uk/preprints/NI03066.pdf).

10. Ярыкин П.Н. Поведение стохастического процесса, описываюшего систему частиц со взаимодействием среднего поля. - Вестн. Моск. ун-та., 2004, № 2, c. $55-58$.

Поступила в редакцию

8.VI.2005

(c) $2006 \mathrm{r}$.

GRANDITS P.*, SUMMER C.**
RISK AVERSE ASYMPTOTICS AND
THE OPTIONAL DECOMPOSITION

Рассматривается задача максимизации ожидаемой полезности для обшей функции полезности на $\mathbf{R}$, когда степень неприятия риска агентом возрастает. Показывается, что предельная стратегия является особой, единственной суперхеджируюшей стратегией - так называемой балансовой стратегией. Исследуется связь с опииональным разложением и классом минимальных хеджируюших стратегий, отисанными в [5].

Ключевые слова и фразы: хеджирование, экспоненщиальная функция полезности, неприятие риска, опциональное ра̇зложение.

1. Introduction. In this paper we consider a financial market with an agent who has to satisfy a contingent claim, e.g., an option, at final time $T$. The agent, who has the possibility of investing in an asset of the underlying financial market, has to decide on a trading strategy and there are, at least, two natural, but different possibilities: utility maximization and superhedging. We will investigate the relationship between these two concepts and show how superhedging can be interpreted as the limit of utility maximization. This interpretation will give some new insight into the notion of superhedging.

For example the notion of superhedging in general does not yield a unique outcome, i.e., there are different superhedging strategies that lead to different terminal wealth at time $T$. But by looking at superhedging as a limit of utility maximization one is naturally led to a special, unique outcome.

Let us assume we are given a finite probability space $\Omega=\left\{\omega_{1}, \ldots, \omega_{N}\right\}$, a finite time horizon $T \in \mathbf{N}$, a filtration $\left(\mathscr{F}_{t}\right)_{t=0,1, \ldots, T}$, and a probability measure $\mathbf{P}$. For finite $\Omega$ we always assume that $\mathbf{P}\left[\omega_{i}\right]>0$ for all $i=1, \ldots, N$. Furthermore we assume, as is typically done in arbitrage pricing theory, that $\mathscr{F}_{0}$ is trivial. The market consists of a stock $S=\left(S_{t}\right)_{t=0,1, \ldots, T}$, i.e., an $\mathbf{R}$-valued adapted process, and a riskless bond $B$. We assume without loss of generality that the stock $S$ represents the discounted price process, i.e., that it is denoted in terms of units of the riskless bond, and that the bond is constant equal to 1 .

The stock process $S$ can be represented by an event tree. Each node in the tree corresponds to an atom $F \in \mathscr{F}_{t}, t=0,1, \ldots, T$. By an atom $F \in \mathscr{F}_{t}$ we mean an element $F \in \mathscr{F}_{t}, F \neq \varnothing$ such that $G \in \mathscr{F}_{t}$ with $G \subsetneq F$ implies $G=\varnothing$. By a slight abuse of notation we say node $F$ at time $t$ for an atom $F \in \mathscr{F}_{t}$. $G$ is an successor of $F \in \mathscr{F}_{t}$, if $G \in \mathscr{F}_{t+1}$ and $G \subset F, G \neq \varnothing$.

* Technische Universität Wien, Karlsplatz, 13, A-1040, Wien, Österreich; e-mail: pgrand@fam.tuwien.ac.at

** Wirtschaftsuniviersität Wien, Institute für Kreditwirtschaft, Nordbergstraße, 15, A-1090 Wien, Österreich; e-mail: csummer@fam.tuwien.ac.at

1) Financial support by the Austrian Nationalbank, Jubiläumsfond 8699 and the Austrian Science Fund (FWF) under grant SFB010 is gratefully acknowledged. 